\title{
SEMI-MARKOV APPROACH TO THE PROBLEM OF DELAYED REFLECTION OF DIFFUSION MARKOV PROCESSES
}

UDC 519.21

\author{
B. P. HARLAMOV
}

\begin{abstract}
A one-dimensional diffusion process taking positive values and reflecting from zero is considered. All the variants of reflecting that preserve of the semi-Markov property are described. This property is characterized by a family of Laplace images of times between the first hitting of zero and first hitting of a level $r$ for any $r>0$. The parameter of this family is used to construct a time change transforming a process with instantaneous reflection to the process with delayed reflection.
\end{abstract}

\section{INTRODUCTION}

I. Gihman and A. Skorohod are the first to investigate the reflection with delay for onedimensional Markov diffusion processes ([1, p. 197]). They applied a method of stochastic integral equations which is based on preserving the Markov property while reflecting. However, there are examples of interactions between a process and a boundary of its range of values, that can be interpreted as reflections, such that the Markov property is being lost, although the semi-Markov property of continuous processes is preserved. Here is a suitable example.

Let $w(t), t \geq 0$, be a Wiener process. Consider the truncated process by a segment $[a, b], a<w(0)<b$,

$$
\bar{w}(t)= \begin{cases}b, & w(t) \geq b, \\ w(t), & a<w(t)<b, \\ a, & w(t) \leq a,\end{cases}
$$

for all $t \geq 0$. It is clear that $\bar{w}(t)$ is not Markovian, however, it is a continuous semiMarkov process [4]: the Markov property holds with respect to the first exit time from any open interval inside the segment, and also with respect to that from any one-sided neighborhood of any end point of the segment.

The semi-Markov approach to the problem of reflection is stated as follows: to determine a semi-Markov transition function for the process at a boundary point if the process preserves its diffusion form inside its open range of values. In other words, if the process preserves its diffusion form at the first exit time from the region and at any time when it leaves the boundary. A more specific problem to find a reflection preserving a global Markov property is reduced to a problem of finding a subclass of Markov reflected processes in the class of all semi-Markov processes. Problems of this kind are important

2010 Mathematics Subject Classification. Primary 60J25, 60J60.

Key words and phrases. Diffusion, Markov property, continuous semi-Markov processes, reflection, delaying, first exit time, transition function, Laplace transform, change of time.

This work was supported by grant RFBR 12-01-00457-a. 
for applications where one takes into account an interaction of diffusion particles with a boundary of a container, leading to a dynamic equilibrium of the system (see, e.g., [7]).

The whole class of semi-Markov characteristics of reflection for a given locally Markov diffusion process is described in 3 . Conditions for a semi-Markov process to be a Markov process are found in [5]. In the present paper, we continue to investigate processes with a semi-Markov reflection. Our aim is to find a formula characterizing a change of time that transforms a process with an instantaneous reflection into a process with a delayed reflection.

While analyzing a two-dimensional diffusion process in a neighborhood of a flat screen, a time change in a tangential component of the process with respect to a time run of a normal component is treated in [6]. This splitting of the process into two components makes the understanding of the situation easier, but at the same time it masks the true mechanism of transformation. In fact, the time change could be discovered on the initial stage of the semi-Markov approach to the problem of reflection. In the present paper, this shortcoming of [6] is removed.

\section{Semi-Markov transition Function on a Boundary}

We consider a diffusion process $X(t)$ on the half-line $t \geq 0$ with the boundary at zero. We assume that the process does not go to infinity and, if started from an arbitrary positive initial point, it hits zero with probability one. For example, this property is satisfied by any diffusion Markov process with a negative drift and bounded local variance. We have explained above why it is expedient to consider semi-Markov reflections. The semi-Markov approach permits us to consider, from a unified point of view, instantaneous reflections as well as truncations.

In the frame of semi-Markov models of reflection, it is natural to assume that $X(t)$ is a semi-Markov process of diffusion type. Let $\left(P_{x}\right), x \geq 0$, be a consistent family of measures generated by the process that depend on initial points of trajectories. On the interval $(0, \infty)$, semi-Markov transition generating functions of the process

$$
\begin{aligned}
& g_{(a, b)}(\lambda, x)=\mathbb{E}_{x}\left(e^{-\lambda \sigma_{(a, b)}} ; \sigma_{(a, b)}<\infty, X\left(\sigma_{(a, b)}=a\right)\right), \\
& h_{(a, b)}(\lambda, x)=\mathbb{E}_{x}\left(e^{-\lambda \sigma_{(a, b)}} ; \sigma_{(a, b)}<\infty, X\left(\sigma_{(a, b)}=b\right)\right),
\end{aligned}
$$

$a<x<b, \lambda>0$, satisfy the differential equation

$$
\frac{1}{2} f^{\prime \prime}+A(x) f^{\prime}-B(\lambda, x) f=0
$$

with the boundary conditions

$$
g_{(a, b)}(\lambda, a+)=h_{(a, b)}(\lambda, b-)=1, \quad g_{(a, b)}(\lambda, b-)=h_{(a, b)}(\lambda, a+)=0 .
$$

The coefficients of the equation are assumed to be piecewise continuous functions of $x>0$. For any $x$, let the function $B(\lambda, x)$ be non-negative and have the completely monotone partial derivative with respect to $\lambda$.

First of all, the reflection of the process from the point $x=0$ means the inclusion of this point to the range of values of the process. Further, we consider semi-closed intervals $[0, r)$ and assume that the process can exit through the endpoint $r$ only. The corresponding semi-Markov transition generating functions are denoted by $h_{[0, r)}(\lambda, x)$, where we admit that $h_{[0, r)}(\lambda, 0)>0$. The function $K(\lambda, r):=h_{[0, r)}(\lambda, 0)$ plays an important role when describing the properties of reflected processes. Using the semi-Markov properties of the process, we obtain

$$
h_{[0, r)}(\lambda, x)=h_{(0, r)}(\lambda, x)+g_{(0, r)}(\lambda, x) K(\lambda, r)
$$


and

$$
K(\lambda, r)=K(\lambda, r-\varepsilon)\left(h_{(0, r)}(\lambda, r-\varepsilon)+g_{(0, r)}(\lambda, r-\varepsilon) K(\lambda, r)\right) .
$$

Assuming that the derivatives with respect to the second argument exist we have

$$
\begin{gathered}
g_{(a, b)}(\lambda, x)=1+g_{(a, b)}^{\prime}(\lambda, a+)(x-a)+o(x-a), \\
g_{(a, b)}(\lambda, x)=-g_{(a, b)}^{\prime}(\lambda, b-)(b-x)+o(b-x), \\
h_{(a, b)}(\lambda, x)=h_{(a, b)}^{\prime}(\lambda, a+)(x-a)+o(x-a), \\
h_{(a, b)}(\lambda, x)=1-h_{(a, b)}^{\prime}(\lambda, b-)(b-x)+o(b-x) .
\end{gathered}
$$

Thus we obtain the differential equation

$$
K^{\prime}(\lambda, r)+K(\lambda, r) h_{(0, r)}^{\prime}(\lambda, r-)+K^{2}(\lambda, r) g_{(0, r)}^{\prime}(\lambda, r-)=0
$$

Its general solution is given by

$$
K(\lambda, r)=\frac{h_{(0, r)}^{\prime}(\lambda, 0+)}{C(\lambda)-g_{(0, r)}^{\prime}(\lambda, 0+)},
$$

where the arbitrary constant $C(\lambda)$ may depend on $\lambda$. In order for $K(\lambda, r)$ to be a Laplace transform it is sufficient that the function $C(\lambda)$ is non-decreasing, $C(0)=0$, and its derivative is a completely monotone function; see [5]. Under our assumptions,

$$
K(\lambda, r)=1-C(\lambda) r+o(r), \quad r \rightarrow 0 .
$$

Our next aim is to find a time change for the process with an instantaneous reflection which transforms it into the process with a delayed reflection.

\section{Time Change With RESPECT TO THE TIME RUN UNDER INSTANTANEOUS REFLECTION}

Denote by $\theta_{t}$ the shift operator acting in the set of trajectories and let $\sigma_{\Delta}$ be the operator of the first exit time from the set $\Delta$. For arbitrary Markov times $\tau_{1}$ and $\tau_{2}$ (considered with respect to the natural filtration), we introduce the operation

$$
\tau_{1} \dot{+} \tau_{2}:=\tau_{1}+\tau_{2} \circ \theta_{\tau_{1}}
$$

on the set $\left\{\tau_{1}<\infty\right\}$. It is known (see [4]) that, for all open (in the relative topology) sets $\Delta_{1}$ and $\Delta_{2}$ with $\Delta_{1} \subset \Delta_{2}$, we have

$$
\sigma_{\Delta_{2}}=\sigma_{\Delta_{1}} \dot{+} \sigma_{\Delta_{2}}
$$

In this case, $\sigma_{\Delta}(\xi)=0$ if $\xi(0) \notin \Delta$.

Below is some further notation for first exit times, their combinations, and for random intervals

$$
\begin{gathered}
\alpha:=\sigma_{[0, \varepsilon)}, \quad \beta:=\sigma_{(0, \infty)}, \quad \gamma(0):=\beta, \\
\gamma:=\alpha \dot{+} \beta, \quad \gamma(n):=\gamma(n-1) \dot{+} \gamma, \quad n \geq 1, \\
b(0):=[0, \beta), \quad a(n):=[\gamma(n-1), \gamma(n-1) \dot{+} \alpha), \quad b(n)=\left[\gamma(n-1) \dot{+} \alpha, \gamma_{n}\right) .
\end{gathered}
$$

The random times $\alpha, \gamma(n)$, and intervals $a(n), b(n), n=1,2, \ldots$, depend on $\varepsilon>0$. Sometimes we denote this dependence by using the corresponding subscript.

Note that $\gamma(n)$ are the moments of jumps of the renewal process. Moreover, if $X(t)>$ 0 , then, for all $t>0$, there exist $\varepsilon>0$ and $n \geq 1$ such that $t \in b_{\varepsilon}(n)$. This implies that, as $\varepsilon \rightarrow 0$, the random set $\bigcup_{k=1}^{\infty} b_{\varepsilon}(k)$ covers the whole set of positive values of the process $X$ with probability one. Possible intervals of constancy and also a discontinuum of points consisting of zeros of process $X$, compose the supplementary set, that is, the limit of the set $\bigcup_{k=1}^{\infty} a_{\epsilon}(k)$ (by definition, discontinuum is a closed set, equivalent to continuum, that 
does not contain any interval, [2, p. 158]). The linear measure of this set is larger than or equal to 0 . This measure is a component of the delay under refection.

It is known (4, p. 111]) that any homogeneous continuous semi-Markov process is a Markov process if and only if there is no intrinsic interval of constancy (it can have an interval of the terminal stopping). This does not imply that a process with delayed reflection cannot have the global Markov property. Its delay is explained completely by the discontinuum. We say that a process has an instantaneous reflection if there is no interval of constancy at zero and if the linear measure of the discontinuum of zeros equals zero.

We are going to construct a sequence of continuous non-decreasing functions $V_{\varepsilon}(t)$, $t \geq 0$, that converge to some limit $V(t)$ as $\varepsilon \rightarrow 0$ uniformly on every bounded interval.

Let $X(0)>0$. Let $V_{\varepsilon}(t)=t$ in the interval $b(0)$ and $V_{\varepsilon}(t)=\beta$ in the interval $a(1)$. Further, the process $V_{\varepsilon}$ increases linearly with the coefficient 1 in the interval $b(1)$, while the function $V_{\varepsilon}$ is a constant in the interval $a(2)$. In interval $b(2), V_{\varepsilon}$ increases with the coefficient 1 . We proceed in the same way and, for every $k$, define $V_{\varepsilon}$ to be a constant in the interval $a(k)$ and to increase with the coefficient 1 in the interval $b(k)$. If $\varepsilon_{1}>\varepsilon_{2}$ for all intervals $a_{\varepsilon_{2}}(k)$, then there exists $n$ such that $a_{\varepsilon_{2}}(k) \subset a_{\varepsilon_{1}}(n)$. Thus we convince ourselves that the functions constructed above are non-decreasing and bounded and consequently approach a limit.

From the original process $X$, we construct a process with an instantaneous reflecting by eliminating all intervals of constancy at zero and by letting the linear measure of its discontinuum of zeros to be zero. This process can be represented as a limit (in the Skorokhod metric) of a sequence of processes $X_{\varepsilon}(t)$ determined for all $t$ as follows:

$$
X_{\varepsilon}(t)=X\left(V_{\varepsilon}^{-1}(t)\right),
$$

where $V_{\varepsilon}^{-1}(y)$ is the first time when the process $V_{\varepsilon}(t)$ hits a level $y$. Hence $X_{\varepsilon}(t)$ has jumps of height $\varepsilon$ at the first hitting time to zero and at its iterations. Denote the process with an instantaneous reflection by $X_{0}(t)$ and the map $X \mapsto X_{0}$ by $\phi_{V}$. The process $X_{0}(t)$ is measurable (with respect to the original $\sigma$-algebra of subsets) and continuous. Let

$$
P_{x}^{0}=P_{x} \circ \phi_{V}^{-1}
$$

be the induced measure of this process.

It is clear that $V$ is the inverse time change transforming the process $X_{0}$ into the process $X$, that is $X=X_{0} \circ V$. In this case, for any open interval $\Delta=(a, b), 0<a<b$, or $\Delta=[0, r), r>0$, we have

$$
\sigma_{\Delta}\left(X_{0} \circ V\right)=V^{-1}\left(\sigma_{\Delta}\left(X_{0}\right)\right) .
$$

The function $V^{-1}$ is called a direct time change corresponding to an arbitrary "intrinsic" Markov time of the original process (to $X_{0}(t)$ in the case under consideration), an analogous time of the transformed process.

Note that, for $\varepsilon_{1}>\varepsilon_{2}$,

$$
\left\{\gamma_{\varepsilon_{1}}(n), n=0,1,2, \ldots\right\}
$$

is a subset of the set

$$
\left\{\gamma_{\varepsilon_{2}}(n), n=0,1,2, \ldots\right\} .
$$

Thus every Markov time $\gamma_{\varepsilon}(n)$ is a Markov regeneration time of the process $V$. This allows us to calculate the finite dimensional distributions of this process. On the other hand, $V$ is completely determined by its inverse

$$
V^{-1}(y):=\inf \{t \geq 0: V(t) \geq y\}, \quad y>0 .
$$


This process is more convenient to deal with, since the Laplace transform of its value at a point $y$ can be found as a limit of a sequence of the Laplace images of values $V_{\varepsilon}^{-1}(y)$ that can be comparatively easy to evaluate.

Theorem 1. A direct time change $V^{-1}(y)$ mapping a process with an instantaneous reflection into a process with delayed reflection satisfies the relation

$$
\mathbb{E}_{0} \exp \left(-\lambda V^{-1}(y)\right)=\mathbb{E}_{0} \exp (-\lambda y-C(\lambda) W(y)),
$$

where $W^{-1}(t)$ is a non-decreasing process with independent increments for which

$$
\mathbb{E}_{0} \exp \left(-\lambda W^{-1}(t)\right)=\exp \left(g_{(0, \infty)}^{\prime}(\lambda, 0+) t\right) .
$$

Short proof. Without loss of generality, we suppose that $X(0)=0$. Let $N_{\varepsilon}(t)=n$ if and only if

$$
\sum_{k=1}^{n-1}|b(k)|<t \leq \sum_{k=1}^{n}|b(k)|,
$$

where $|a(k)|$ and $|b(k)|$ are the lengths of the intervals $a(k)$ and $b(k)$, respectively. Then

$$
\begin{aligned}
\mathbb{E}_{0} \exp \left(-\lambda V^{-1}(y)\right) & =\lim _{\varepsilon \rightarrow 0} \mathbb{E}_{0} \exp \left(-\lambda V_{\varepsilon}^{-1}(y)\right) \\
& =\lim _{\varepsilon \rightarrow 0} \mathbb{E}_{0}\left(-\lambda y-\lambda \sum_{k=1}^{N_{\varepsilon}(y)}|a(k)|\right) .
\end{aligned}
$$

We have

$$
\begin{aligned}
& \mathbb{E}_{0} \exp \left(-\lambda\left(V_{\varepsilon}^{-1}(y)-y\right)\right)=\mathbb{E}_{0} \exp \left(-\lambda \sum_{k=1}^{N_{\varepsilon}(y)}|a(k)|\right) \\
&=\sum_{n=0}^{\infty} \mathbb{E}_{0} \exp \left(-\lambda \sum_{k=1}^{n} \alpha \circ \theta_{\gamma(k-1)} ; N_{\varepsilon}(t)=n\right) \\
&=P_{\varepsilon}(\beta \geq y)+\sum_{n=1}^{\infty} \mathbb{E}_{0}\left(\exp \left(-\lambda \sum_{k=1}^{n} \alpha \circ \theta_{\gamma(k-1)}\right) ; \sum_{k=1}^{n-1}|b(k)|<y \leq \sum_{k=1}^{n}|b(k)|\right) \\
&=P_{\varepsilon}(\beta \geq y) \\
&+\sum_{n=1}^{\infty} \mathbb{E}_{0}\left(\exp \left(-\lambda \alpha-\lambda \sum_{k=2}^{n} \alpha \circ \theta_{\gamma(k-1)}\right) ; \beta \circ \theta_{\alpha}+\sum_{k=2}^{n-1} \beta \circ \theta_{\alpha} \circ \theta_{\gamma(k-1)}<y \leq \beta \circ \theta_{\alpha}+\sum_{k=2}^{n} \beta \circ \theta_{\alpha} \circ \theta_{\gamma(k-1)}\right) \\
&= P_{\varepsilon}(\beta \geq y)+\sum_{n=1}^{\infty} \int_{0}^{y} \mathbb{E}_{0}\left(\exp \left(-\lambda \alpha-\lambda \sum_{k=2}^{n} \alpha \circ \theta_{\gamma(k-1)}\right) ; \beta \circ \theta_{\alpha} \in d x,\right. \\
&\left.\sum_{k=2}^{n-1} \beta \circ \theta_{\alpha} \circ \theta_{\gamma(k-1)}<y-x \leq \sum_{k=2}^{n} \beta \circ \theta_{\alpha} \circ \theta_{\gamma(k-1)}\right)
\end{aligned}
$$




$$
\begin{aligned}
& =P_{\varepsilon}(\beta \geq y) \\
& +\sum_{n=1}^{\infty} \int_{0}^{y} \mathbb{E}_{0}\left(e^{-\lambda \alpha} ; \beta \circ \theta_{\alpha} \in d x\right) \\
& \times \mathbb{E}_{0}\left(\exp \left(-\lambda \sum_{k=2}^{n} \alpha \circ \theta_{\gamma(k-2)}\right) ;\right. \\
& \left.\sum_{k=2}^{n-1} \beta \circ \theta_{\alpha} \circ \theta_{\gamma(k-2)}<y-x \leq \sum_{k=2}^{n} \beta \circ \theta_{\alpha} \circ \theta_{\gamma(k-2)}\right) \\
& =P_{\varepsilon}(\beta \geq y) \\
& +\sum_{n=1}^{\infty} \int_{0}^{y} P_{\varepsilon}(\beta \in d x) \mathbb{E}_{0}\left(e^{-\lambda \alpha}\right) \\
& \times \mathbb{E}_{0}\left(\exp \left(-\lambda \sum_{k=1}^{n-1} \alpha \circ \theta_{\gamma(k-1)}\right) ;\right. \\
& \left.\sum_{k=1}^{n-2} \beta \circ \theta_{\alpha} \circ \theta_{\gamma(k-1)}<y-x \leq \sum_{k=1}^{n-1} \beta \circ \theta_{\alpha} \circ \theta_{\gamma(k-1)}\right) \\
& =P_{\varepsilon}(\beta \geq y) \\
& +\int_{0}^{y} P_{\varepsilon}(\beta \in d x) \mathbb{E}_{0}\left(e^{-\lambda \alpha}\right) \sum_{n=0}^{\infty} \mathbb{E}_{0}\left(\exp \left(-\lambda \sum_{k=1}^{n} \alpha \circ \theta_{\gamma(k-1)}\right) ; N_{\varepsilon}(y-x)=n\right) \\
& =P_{\varepsilon}(\beta \geq y)+\int_{0}^{y} P_{\varepsilon}(\beta \in d x) \mathbb{E}_{0}\left(e^{-\lambda \alpha}\right) \mathbb{E}_{0} \exp \left(-\lambda\left(V_{\varepsilon}^{-1}(y-x)-(y-x)\right)\right) .
\end{aligned}
$$

Put $Z(y):=\mathbb{E}_{0} \exp \left(-\lambda\left(V_{\varepsilon}^{-1}(y)-y\right)\right), F(x):=P_{x}(\beta<x), \bar{F}(x):=1-F(x)$, and $A:=\mathbb{E}_{0}\left(e^{-\lambda \alpha}\right)$. The solution of the integral equation

$$
Z(y)=\bar{F}(x)+A \int_{0}^{y} Z(y-x) d F(x)
$$

can be written as

$$
Z(y)=\sum_{n=0}^{\infty} A^{n}\left(F^{(n)}(y)-F^{(n+1)}(y)\right),
$$

where $F^{(n)}$ is the $n$-times convolution of the distribution $F$. Consider a sequence of independent and identically distributed random values $|b(n)|, n=1,2, \ldots$ Let $P_{\varepsilon}^{*}$ be the distribution of the renewal process $N_{\varepsilon}(y)$ for which $|b(n)|$ is the length of the corresponding interval and $\mathbb{E}_{\varepsilon}^{*}$ is the corresponding expectation. Then

$$
\mathbb{E}_{\varepsilon}^{*} A^{N_{\varepsilon}(y)}=\sum_{n=0}^{\infty} A^{n} P_{\varepsilon}^{*}\left(N_{\varepsilon}(y)=n\right)=\sum_{n=0}^{\infty} A^{n}\left(F^{(n)}(y)-F^{(n+1)}(y)\right) .
$$

Thus

$$
\mathbb{E}_{0} \exp \left(-\lambda V_{\varepsilon}^{-1}(y)\right)=e^{-\lambda y} \mathbb{E}_{\varepsilon}^{*}\left(\mathbb{E}_{0} e^{-\lambda \alpha}\right)^{N_{\varepsilon}(y)} .
$$

On the other hand, it is clear that there exists a version of the process $N_{\varepsilon}(y)$, being measurable with respect to the basic $\sigma$-algebra, which is adapted to the natural filtration of the original process and has the identical distribution with respect to the measure $P_{0}$. Using the above notation,

$$
\mathbb{E}_{\varepsilon}^{*}\left(\mathbb{E}_{0} e^{-\lambda \alpha}\right)^{N_{\varepsilon}(y)}=\mathbb{E}_{0}\left(\mathbb{E}_{0} e^{-\lambda \alpha}\right)^{N_{\varepsilon}(y)} .
$$


Moreover, the measures $P_{0}$ and $P_{0}^{0}$ coincide on the $\sigma$-algebra $F^{*}$ generated by the random values $\beta^{\varepsilon} \circ \theta_{\alpha^{\varepsilon}} \circ \theta_{\gamma(k)^{\varepsilon}}, \varepsilon>0, k=1,2, \ldots$ Thus

$$
\mathbb{E}_{0}\left(\mathbb{E}_{0} e^{-\lambda \alpha}\right)^{N_{\varepsilon}(y)}=\mathbb{E}_{0}^{0}\left(\mathbb{E}_{0} e^{-\lambda \alpha}\right)^{N_{\varepsilon}(y)} .
$$

Since $\alpha$ depends on $\varepsilon$,

$$
\mathbb{E}_{0} e^{-\lambda \alpha}=K(\lambda, \varepsilon)=1-C(\lambda) \varepsilon+o(\varepsilon) .
$$

We show that the process $W_{\varepsilon}(y):=\varepsilon N_{\varepsilon}(y)$ approaches weakly the limit $W(y)$ as $\varepsilon \rightarrow 0$. Note that $W(y)$ is an inverse process with independent increments, measurable with respect to the $\sigma$-algebra $F^{*}$. Note also that the parameters of $W(y)$ are known. The process $W_{\varepsilon}(y)$ is non-decreasing and is characterized completely by the process $W_{\varepsilon}^{-1}(t)$. The latter has independent positive jumps on the lattice with step $\varepsilon$. Hence it is a process with independent increments. It is clear that the limit of a sequence of such processes, if it exists, is a process with independent increments, too. The existence of the limit follows from the Laplace transform of its increments. We have

$$
\begin{aligned}
\mathbb{E}_{0}^{0} e^{-\lambda W_{\varepsilon}^{-1}(t)} & =\mathbb{E}_{0}^{0} \exp \left(-\lambda \sum_{k=1}^{[t / \varepsilon]}|b(k)|\right)=\left(\mathbb{E}_{\varepsilon} e^{-\lambda \beta}\right)^{[t / \varepsilon]} \\
& =\left(1+g_{(0, \infty)}^{\prime}(\lambda, 0) \varepsilon+o(\varepsilon)\right)^{[t / \varepsilon]} \rightarrow e^{g_{(0, \infty)}^{\prime}(\lambda, 0) t}, \quad \varepsilon \rightarrow 0 .
\end{aligned}
$$

Using a sufficient condition for the weak convergence of processes in terms of convergence of their first exit times from open sets ([4], p. 287), we obtain

$$
\mathbb{E}_{0} \exp \left(-\lambda V^{-1}(y)\right)=\mathbb{E}_{0}^{0} \exp (-\lambda y-C(\lambda) W(y)) .
$$

The latter can be considered as a description of the direct time change in terms of the process with an instantaneous reflection and the delay function $C(\lambda)$.

We use this formula for deriving the Laplace transform for the difference between the first exit times from a one-sided neighborhood of the boundary point for the processes with delayed and instantaneous reflections.

Put

$$
\begin{gathered}
\beta^{r}:=\sigma_{(0, r)}, \quad \gamma^{r}(0)=0, \\
\gamma^{r}:=\alpha \dot{+} \beta^{r}, \quad \gamma^{r}(n):=\gamma^{r}(n-1) \dot{+} \gamma^{r}, \quad n \geq 1, \\
b^{r}(n)=\left[\gamma^{r}(n-1) \dot{+} \alpha, \gamma^{r}(n)\right), \quad n \geq 1, \\
M_{\varepsilon}^{r}:=\inf \left\{n \geq 0: X\left(\gamma^{r}(n)\right) \geq r\right\} .
\end{gathered}
$$

Then

$$
\begin{gathered}
P_{0}\left(M_{\varepsilon}^{r}=n\right)=P_{0}\left(X\left(\gamma^{r}(1)\right)=0, \ldots, X\left(\gamma^{r}(n-1)\right)=0,\right. \\
\left.X\left(\gamma^{r}(n-1)\right)=r\right)=(p(\varepsilon, r))^{n-1}(1-p(\varepsilon, r)),
\end{gathered}
$$

where $p(\varepsilon, r):=P_{0}\left(X\left(\gamma^{r}(1)\right)=0\right)$.

Theorem 2. The difference between the first exit times from the interval $[0, r)$ for the processes with delayed and instantaneous reflections is such that

$$
\mathbb{E}_{0} \exp \left(-\lambda\left(\sigma_{[0, r)}-\sigma_{[0, r)}^{0}\right)\right)=\frac{-G_{(0, r)}^{\prime}(0+)}{C(\lambda)-G_{(0, r)}^{\prime}(0+)},
$$

where $G_{(0, r)}(x)=g_{(0, r)}(0, x)$. 
Short proof. Let $X(0)=0$. Then $\sigma_{[0, r)}=\gamma_{M_{\varepsilon}^{r}}^{r}$ for all $\varepsilon<r$. On the other hand, $\gamma^{r}=\gamma$ in the set $\left\{X\left(\gamma^{r}\right)=0\right\}$. We conclude by induction that

$$
\gamma^{r}(n)=\gamma(n) \quad \text { in the set } \bigcap_{k=1}^{n}\left\{X\left(\gamma^{r}(k)\right)=0\right\}
$$

whence

$$
\begin{aligned}
\gamma^{r}(n) I\left(M_{\varepsilon}^{r}=n\right) & =\left(\gamma^{r}(n-1) \dot{+} \gamma^{r}\right) I\left(\bigcap_{k=1}^{n-1}\left\{X\left(\gamma^{r}(k)\right)=0\right\}\right) \cap\left\{X\left(\gamma^{r}(n)\right)=r\right\} \\
& =\left(\gamma(n-1) \dot{+} \gamma^{r}\right) I\left(M_{\varepsilon}^{r}=n\right) .
\end{aligned}
$$

Let $\sigma_{[0, r)}^{0}$ be the first exit time from the interval $[0, r)$ of the process with an instantaneous reflection (formally, this means that $\left.\sigma_{[0, r)}^{0}=V\left(\sigma_{[0, r)}\right)\right)$. Then $V^{-1}\left(\sigma_{[0, r)}^{0}\right)=\sigma_{[0, r)}$ and (11) implies

$$
\begin{aligned}
\mathbb{E}_{0} \exp \left(-\lambda\left(\sigma_{[0, r)}-\sigma_{[0, r)}^{0}\right)\right)=\mathbb{E}_{0}^{0} \exp \left(-C(\lambda) W\left(\sigma_{[0, r)}^{0}\right)\right) \\
=\mathbb{E}_{0}^{0} \exp \left(-C(\lambda) W\left(\gamma^{r}\left(M_{\varepsilon}^{r}\right)\right)\right)=\sum_{n=1}^{\infty} \mathbb{E}_{0}^{0}\left(\exp \left(-C(\lambda) W\left(\gamma^{r}(n)\right)\right) ; M_{\varepsilon}^{r}=n\right) \\
=\sum_{n=1}^{\infty} \mathbb{E}_{0}^{0} \exp \left(-C(\lambda) W\left(\gamma(n-1) \dot{+} \gamma^{r}\right) ; M_{\varepsilon}^{r}=n\right) \\
=\sum_{n=1}^{\infty} \mathbb{E}_{0}^{0}\left(\exp \left(-C(\lambda) W\left(\sum_{k=1}^{n-1}\left(|a(k)|+\left|b^{r}(k)\right|\right)+|a(n)|+\left|b^{r}(n)\right|\right) ; M_{\varepsilon}^{r}=n\right)\right.
\end{aligned}
$$

Since $\sum_{k=1}^{M_{\varepsilon}^{r}}(|a(k)|) \rightarrow 0$ almost surely as $\varepsilon \rightarrow 0$ with respect to $P_{0}^{0}$, we have

$$
\begin{aligned}
\lim _{\varepsilon \rightarrow 0} & \sum_{n=1}^{\infty} \mathbb{E}_{0}^{0}\left(\exp (-C(\lambda)) W\left(\sum_{k=1}^{n-1}\left(|a(k)|+\left|b^{r}(k)\right|\right)+|a(n)|+\left|b^{r}(n)\right|\right) ; M_{\varepsilon}^{r}=n\right) \\
& =\lim _{\varepsilon \rightarrow 0} \mathbb{E}_{0}^{0}\left(\exp (-C(\lambda)) W\left(\sum_{k=1}^{M_{\varepsilon}^{r}-1}\left|b^{r}(k)\right|+\left|b^{r}\left(M_{\varepsilon}^{r}\right)\right|\right)\right) \\
& =\lim _{\varepsilon \rightarrow 0} \mathbb{E}_{0}^{0}\left(\exp (-C(\lambda)) \varepsilon N_{\varepsilon}\left(\sum_{k=1}^{M_{\varepsilon}^{r}-1}\left|b^{r}(k)\right|+\left|b^{r}\left(M_{\varepsilon}^{r}\right)\right|\right)\right) .
\end{aligned}
$$

The definition of the process $N_{\varepsilon}(t)$ implies that

$$
N_{\varepsilon}\left(\sum_{k=1}^{n-1}\left|b^{r}(k)\right|+\left|b^{r}(n)\right|\right)=n, \quad n=1,2 \ldots .
$$

Therefore

$$
\begin{aligned}
\mathbb{E}_{0} \exp \left(-\lambda\left(\sigma_{[0, r)}-\sigma_{[0, r)}^{0}\right)\right) & =\lim _{\varepsilon \rightarrow 0} \mathbb{E}_{0}^{0} \exp \left(-C(\lambda) \varepsilon M_{\varepsilon}^{r}\right) \\
& =\lim _{\varepsilon \rightarrow 0} \sum_{n=1}^{\infty} e^{-C(\lambda) \varepsilon n}(p(\varepsilon, r))^{n-1}(1-p(\varepsilon, r)) \\
& =\lim _{\varepsilon \rightarrow 0} e^{-C(\lambda) \varepsilon} \frac{1-p(\varepsilon, r)}{1-e^{-C(\lambda) \varepsilon} p(\varepsilon, r)}
\end{aligned}
$$


Taking into account that

$$
\begin{aligned}
p(\varepsilon, r) & =P_{0}\left(X\left(\gamma_{\varepsilon}^{r}\right)=0\right)=P_{0}\left(X\left(\alpha_{\varepsilon} \dot{+} \beta_{\varepsilon}^{r}\right)=0\right)=P_{0}\left(X\left(\beta_{\varepsilon}^{r}\right) \circ \theta_{\alpha_{\varepsilon}}=0\right) \\
& =P_{\varepsilon}\left(X\left(\beta_{\varepsilon}^{r}\right)=0\right):=G_{(0, r)}(\varepsilon)
\end{aligned}
$$

and that the right hand side (the value of $g_{(0, r)}(\lambda, \varepsilon)$ for $\lambda=0$ ) is such that

$$
G_{(0, r)}(\varepsilon)=1+G_{(0, r)}^{\prime}(0+) \varepsilon+o(\varepsilon),
$$

we finally obtain

$$
\mathbb{E}_{0} \exp \left(-\lambda\left(\sigma_{[0, r)}-\sigma_{[0, r)}^{0}\right)\right)=\frac{-G_{(0, r)}^{\prime}(0+)}{C(\lambda)-G_{(0, r)}^{\prime}(0+)} .
$$

Note that if $C(\lambda)=k \lambda$ (in which case the reflecting locally Markov process is globally Markov [5]), the difference between the first exit times from the interval $[0, r)$ for the processes with delayed and instantaneous reflections has the exponential distribution with parameter $-G_{(0, r)}^{\prime}(0+) / k$.

\section{EXAmple}

Consider the truncated standard Wiener process

$$
\bar{w}(t)= \begin{cases}0, & w(t) \leq 0 \\ w(t), & w(t)>0\end{cases}
$$

In the framework of the semi-Markov model of reflection, $\bar{w}(t)$ is characterized by the function

$$
K(\lambda, r)=\frac{h_{(0, r)}^{\prime}(\lambda, 0+)}{C(\lambda)-g_{(0, r)}^{\prime}(\lambda, 0+)}=\frac{\sqrt{2 \lambda} / \sinh r \sqrt{2 \lambda}}{C(\lambda)-\sqrt{2 \lambda} \cosh r \sqrt{2 \lambda} / \sinh r \sqrt{2 \lambda}} .
$$

Taking into account the definition of this process one can write

$$
K(\lambda, r)=\mathbb{E}_{0}^{w} \exp \left(-\lambda \sigma_{(-\infty, r)}\right)=\exp (-r \sqrt{2 \lambda}) .
$$

Comparing the derivatives at zero of these two representations of the same function, we obtain $C(\lambda)=\sqrt{2 \lambda}$. Now we can evaluate the main characteristic of delay for this process under reflection (including the lengths of all intervals of constancy) between the first hitting time of the level 0 and the first hitting time of the level $r$ :

$$
\mathbb{E}_{0} \exp \left(-\lambda\left(\sigma_{[0, r)}-\sigma_{[0, r)}^{0}\right)\right)=\frac{1 / r}{\sqrt{2 \lambda}+1 / r} .
$$

The latter result can be found in the list of known Laplace transforms, so we do not provide its proof.

\section{BIBLIOGRAPHY}

1. I. I. Gihman and A. V. Skorokhod, Stochastic Differential Equation, "Naukova Dumka", Kiev, 1968; English transl. Springer-Verlag, Berlin, 1972. MR.0346904 (49 \#11625)

2. F. Hausdorff, Set Theory, Chelsea Publishing Co., New York, 1962. (translated from the German) MR0141601 (25:4999)

3. B. P. Harlamov, Diffusion processes with delay at the endpoints of a segment, Zapiski Nauchnyh Seminarov POMI 351 (2007), 284-297; English transl. in J. Math. Sci. 152 (2008), no. 6, 958965. MR2742915(2011j:60267)

4. B. P. Harlamov, Continuous Semi-Markov Processes, Applied Stochastic Methods Series. ISTE, London; John Wiley \& Sons, Inc., Hoboken, NJ, 2008. MR2376500 (2009b:60275)

5. B. P. Harlamov, On Markov diffusion processes with delayed reflection from boundaries of a segment, Zapiski Nauchnyh Seminarov POMI 368 (2009), 243-267; English transl. in J. Math. Sci. 167, (2010), no. 4, 574-587. MR2749196(2012b:60261) 
6. S. S. Rasova and B. P. Harlamov, On motion of Brownian particles along a delaying screen, Zapiski Nauchnyh Seminarov POMI 396 (2011), 175-194; English transl. in J. Math. Sci. 188 (2013), no. 6, 737-747. MR2870140

7. B. P. Harlamov, Stochastic model of gas capillary chromatography, Comm. Statist. Simulation Comput. 41 (2012), no. 7, 1023-1031. MR2912932

Institute of Problems of Mechanical Engineering, RAS, Saint-Petersburg, Russia

E-mail address: b.p.harlamov@gmail.com

Received 30/JAN/2013

Originally published in English 